\title{
GUARANTEED NONLINEAR STATE ESTIMATION FOR CONTINUOUS-TIME DYNAMICAL MODELS FROM DISCRETE-TIME MEASUREMENTS
}

\author{
Michel Kieffer Eric Walter \\ Laboratoire des Signaux et Systèmes \\ CNRS - Supélec - Université Paris-Sud \\ Plateau de Moulon, F-91192 Gif-sur-Yvette, France \\ \{kieffer,walter\}@lss.supelec.fr
}

\begin{abstract}
This paper is about bounded-error state estimation for models described by a continuous-time state equation from discrete-time measurements. A guaranteed solution to this problem is proposed, based on Müller's theorems and interval analysis. This technique allows, at any time instant, the characterization of the set of all state vectors that are consistent with the error bounds, measurements and model structure. Joint state and parameter estimation is also possible. The resulting methodology is illustrated on the estimation of the state of a compartmental model.
\end{abstract}

Keywords: Bounded-error estimation, continuous-time models, guaranteed estimation, interval analysis, state estimation

\section{INTRODUCTION}

This paper is about guaranteed state estimation for systems described by nonlinear continuous-time dynamical models of the form

$$
\mathbf{x}^{\prime}(t)=\frac{\mathrm{d} \mathbf{x}}{\mathrm{d} t}(t)=\mathbf{f}(\mathbf{x}(t), \mathbf{p}, \mathbf{v}(t), t), \mathbf{x}\left(t_{0}\right)=\mathbf{x}_{0},
$$

where $\mathbf{x} \in \mathbb{D} \subset \mathbb{R}^{n_{\mathrm{x}}}$ is the state of the model with initial condition $\mathbf{x}_{0}$ at $t_{0}, \mathbf{p} \in \mathbb{R}^{n_{\mathrm{p}}}$ is a parameter vector and $\mathbf{v}(t)$ is some state perturbation taking into account the fact that the model is only an approximation of reality. For the sake of brevity, the dependency in $t$ of $\mathbf{x}$ and $\mathbf{x}^{\prime}$ will be omitted in what follows. We assume that measurements $\mathbf{y}\left(t_{k}\right) \in \mathbb{R}^{n_{\mathrm{y}}}$ are taken at discrete time instants denoted by $t_{k}$, with $k=1, \ldots, N$ and such that $t_{k+1}>t_{k} \geqslant t_{0}$. The measurement process is assumed described by the model

$$
\mathbf{y}_{\mathrm{m}}\left(\mathbf{p}, t_{k}\right)=\mathbf{h}\left(\mathbf{x}\left(t_{k}\right), \mathbf{p}, \mathbf{w}\left(t_{k}\right), t_{k}\right),
$$

where $\mathbf{w}\left(t_{k}\right)$ is the measurement noise at time $t_{k}$.

In this paper, state estimation is considered in a bounded-error context under the following assumptions. The initial state $\mathbf{x}\left(t_{0}\right)$ belongs to a box (or interval vector) $\left[\mathbf{x}_{0}\right]=\left[\underline{\mathbf{x}}_{0}, \overline{\mathbf{x}}_{0}\right]$ and for all $t \in\left[t_{0}, t_{N}\right]$, $\mathbf{x}(t)$ remains in some compact set $\mathbb{D} \subset \mathbb{R}^{n_{\mathrm{x}}}$. The parameter vector $\mathbf{p}$ is constant and belongs to $\left[\mathbf{p}_{0}\right]=$ $\left[\underline{\mathbf{p}}_{0}, \overline{\mathbf{p}}_{0}\right]$ (this assumption is not essential and could be relaxed by incorporating $\mathbf{p}$ in some extended state vector, provided that a model is chosen for the evolution of $\mathbf{p}$ ). The state perturbation $\mathbf{v}(\cdot)$ belongs to $\mathcal{V}$, the set of all continuous functions such that

$$
\underline{\mathbf{v}}(t) \leqslant \mathbf{v}(t) \leqslant \overline{\mathbf{v}}(t) \text { for any } t \in\left[t_{0}, t_{N}\right],
$$

with $\underline{\mathbf{v}}(t)$ and $\overline{\mathbf{v}}(t)$ known for all $t \in\left[t_{0}, t_{N}\right]$. The measurement noise $\mathbf{w}\left(t_{k}\right)$ belongs to $\left[\mathbf{w}\left(t_{k}\right)\right]=$ $\left[\underline{\mathbf{w}}\left(t_{k}\right), \overline{\mathbf{w}}\left(t_{k}\right)\right]$, which is known for all $t_{k}$. The available information at time $t \geqslant t_{0}$ is

$$
\begin{aligned}
\mathcal{I}(t)= & \left\{\left[\mathbf{x}_{0}\right],\left[\mathbf{p}_{0}\right],\{[\mathbf{v}(\tau)]\}_{\tau \in\left[t_{0}, t\right]},\right. \\
& \left.\left\{\mathbf{y}\left(t_{k}\right),\left[\mathbf{w}\left(t_{k}\right)\right]\right\}_{k=1}^{M}\right\},
\end{aligned}
$$

where $t_{M}$ is such that $t_{M} \leqslant t<t_{M+1}$. Causal set estimation of the state then amounts to the characterization of the set $\mathbb{X}_{t \mid t}$ of all values of $\mathbf{x}(t)$ that are consistent with $\mathcal{I}(t)$ for any given $t \geqslant t_{0}$.

In this context, the state of the art is much less advanced than for discrete-time models, which is unfortunate as continuous-time knowledge-based models appear in countless potential applications, for instance in physics, chemistry, and the biosciences. When the state perturbation and measurement noise are both assumed to belong to some known compact sets, $\mathbf{p}$ is known (or equivalently there are no parameters to be 
estimated) and $\mathbf{f}$ and $\mathbf{h}$ are linear in $\mathbf{x}$, algorithmic tools are available to compute ellipsoids guaranteed to contain the state vector at any given instant of time, see, e.g., (Chernousko, 1994; Kurzhanski and Valyi, 1997; Durieu et al., 2001).

When $\mathbf{f}$ or $\mathbf{h}$ are nonlinear in $\mathbf{x}$, techniques making it possible to bracket the state vector between some lower and upper vectors that can be computed at any given instant of time under some hypotheses on the signs of the entries of the Jacobian matrix $\partial \mathbf{f} / \partial \mathbf{x}$ have been described in the context of waste-water treatment applications in (Gouzé et al., 2000) and (Rapaport and Gouzé, 2003). The resulting state estimator has been called an interval observer. Under the same hypotheses, and using these results and those obtained in discrete-time state estimation (Kieffer and Walter, 2005) proposes to replace the box-enclosure of $\mathbb{X}_{t \mid t}$ by an enclosure in a potentially much more precise union of non-overlapping boxes.

In a more general context, a recursive state estimation algorithm based on the guaranteed numerical integration of ordinary differential equations (ODEs) (Berz and Makino, 1998; Nedialkov and Jackson, 2001) has been presented in (Jaulin, 2002), for the case where there are no state perturbations. This technique has been improved in (Raissi et al., 2004), using results taken from (Lohner, 1992; Nedialkov and Jackson, 2001) and applied to the problem of joint parameter and state estimation. However, both techniques have difficulties to cope with highly uncertain initial conditions and state perturbations.

The results described in (Gouzé et al., 2000) were generalized using Müller's theorems (Müller, 1926) in (Gennat and Tibken, 2004) and applied to system simulation, with no condition on the entries of $\partial \mathbf{f} / \partial \mathbf{x}$ ( $\mathrm{f}$ is even not assumed differentiable with respect to $\mathrm{x})$.

The aim of this paper is to show how Müller's theorems can be employed for continuous-time state estimation. An idealized state-estimation algorithm will be briefly recalled in Section 2. How interval analysis can be used to implement this idealized algorithm will be sketched in Section 3. A key requirement is the ability to enclose the solution of (1) between those of two bounding systems. A solution using Müller's theorems will be presented in Section 4, before some illustrative examples in Section 5.

\section{IDEALIZED ALGORITHM}

An idealized algorithm similar to that described for instance in (Jaulin et al., 2001) may be built. As a Kalman filter, it alternates prediction and correction steps. For the prediction step, if $\mathbb{X}_{t_{k} \mid t_{k}}$ is the set of all values of the state vector $\mathbf{x}\left(t_{k}\right)$ that are consistent with $\mathcal{I}\left(t_{k}\right)$, then it should in principle be possible to compute $\mathbb{X}_{t_{k+1} \mid t_{k+1}}$ as follows.

For any $\mathbf{x} \in \mathbb{X}_{t_{k} \mid t_{k}}$, let $\varphi\left(\mathbf{x}, t, t_{k}, \mathbf{p},\{\mathbf{v}(\tau)\}_{\tau \in\left[t_{k}, t\right]}\right)$ be the value at time $t$ of the solution of (1) that coincides with $\mathrm{x}$ at time $t_{k}$. The predicted set estimate $\mathbb{X}_{t_{k+1} \mid t_{k}}$ at time $t_{k+1}$ may then be written as

$$
\begin{aligned}
\mathbb{X}_{t_{k+1} \mid t_{k}}= & \left\{\varphi\left(\mathbf{x}, t_{k+1}, t_{k}, \mathbf{p},\{\mathbf{v}(\tau)\}_{\tau \in\left[t_{k}, t_{k+1}\right]}\right)\right. \\
& \mid \mathbf{p} \in\left[\mathbf{p}_{0}\right], \mathbf{v}(\tau) \in[\mathbf{v}(\tau)], \mathbf{x} \in \mathbb{X}_{t_{k} \mid t_{k}}, \\
& \left.\tau \in\left[t_{k}, t_{k+1}\right]\right\} .
\end{aligned}
$$

By construction, $\mathbf{x}\left(t_{k+1}\right) \in \mathbb{X}_{t_{k+1} \mid t_{k}}$.

The corrected set estimate could in principle be obtained by taking advantage of the information contained in $\mathbf{y}\left(t_{k+1}\right)$, the system output measured at time $t_{k+1}$, as follows

$$
\begin{aligned}
\mathbb{X}_{t_{k+1} \mid t_{k+1}}= & \left\{\mathbf{x} \in \mathbb{X}_{t_{k+1} \mid t_{k}} \mid \mathbf{y}\left(t_{k+1}\right) \in\right. \\
& \left.\mathbf{h}\left(\mathbf{x},\left[\mathbf{p}_{0}\right],\left[\mathbf{w}\left(t_{k+1}\right)\right], t_{k+1}\right)\right\} .
\end{aligned}
$$

Provided that all hypotheses are satisfied, $\mathbb{X}_{t_{k+1} \mid t_{k+1}}$ does contain $\mathbf{x}\left(t_{k+1}\right)$.

\section{INTERVAL ANALYSIS}

Interval analysis, see, e.g., (Jaulin et al., 2001) provides tools for computing with sets, which are described using outer-approximations formed by union of non-overlapping boxes (subpavings). It is, for instance, possible to characterize in an approximate but guaranteed way ${ }^{1}$

- the inverse image of a set by a given function using the SIVIA algorithm (Set Inverter Via Interval Analysis) (Jaulin and Walter, 1993; Jaulin et al., 2001)

- the direct image of a set by a given function using the IMAGESP algorithm (Image Subpaving) (Kieffer et al., 2002).

Both algorithms require an inclusion function for the function used for direct or inverse image evaluation. An interval inclusion function $[f](\cdot)$ for a real function $f: \mathcal{D} \subset \mathbb{R} \rightarrow \mathbb{R}$ is an interval-valued function, such that for any interval $[x] \subset \mathcal{D}, f([x]) \subset[f]([x])$, where $f([x])=\{f(x), x \in[x]\}$; see (Jaulin et $a l ., 2001)$ for more details, including the extension to vector functions.

For the correction step, an inclusion function for $\mathbf{h}$ is usually easily obtained. If one uses the representation of compact sets by subpavings, the correction step poses no specific difficulty, and may be implemented in an approximate but guaranteed way by using SIVIA.

The main difficulty lies in the prediction step to evaluate $\mathbb{X}_{t_{k+1} \mid t_{k}}$, which may be viewed as the direct image of a set by the function $\varphi$. An inclusion function for $\varphi$ is much more difficult to obtain. Guaranteed numerical integration (Lohner, 1992; Berz and Makino, 1998; Nedialkov and Jackson, 2001) was designed to integrate deterministic ODEs while taking into account numerical errors, and often gives very disappointing results when there are uncertain initial

\footnotetext{
1 Guaranteed means here that what is computed is an outer approximation of the actual set of interest
} 
conditions or parameters and in the presence of state perturbations. In this case, the outer approximations of the predicted set $\overline{\mathbb{X}}_{t_{k+1} \mid t_{k}}$ that might thus be obtained may turn out to be too pessimistic to be of any practical use.

\section{GUARANTEED NUMERICAL ENCLOSURES FOR DIFFERENTIAL MODELS}

One way to bypass the difficulty mentioned in the previous section for specific but significant classes of models is to bracket all solutions of the uncertain state equation (1) between those of deterministic lower and upper dynamical systems.

A first class of models for which this is easy is when, as in (Gouzé et al., 2000) and (Rapaport and Gouzé, 2003), (1) is cooperative, that is to say it belongs to a specific class of dynamical systems preserving an order relation in state space.

Definition 1. The system $\mathbf{x}^{\prime}=\mathbf{f}(\mathbf{x}, \mathbf{p}, \mathbf{v}, t)$ is cooperative over $\mathbb{D} \times\left[\underline{\mathbf{p}}_{0}, \overline{\mathbf{p}}_{0}\right] \times \mathcal{V} \times\left[t_{0}, t_{N}\right]$ if for any $i=1 \ldots n$, the fact that $\mathbf{x} \in \mathbb{D}$ and $\mathbf{y} \in \mathbb{D}$ with

$$
x_{j} \leqslant y_{j}, j \neq i
$$

implies

$$
\begin{aligned}
& f_{i}\left(x_{1}, \ldots, x_{i-1}, x_{i}, x_{i+1}, \ldots x_{n_{\mathrm{x}}}, \mathbf{p}, \mathbf{v}, t\right) \\
& \quad \leqslant f_{i}\left(y_{1}, \ldots, y_{i-1}, x_{i}, y_{i+1}, \ldots y_{n_{\mathrm{x}}}, \mathbf{p}, \mathbf{v}, t\right)
\end{aligned}
$$

for any $\mathbf{p} \in\left[\underline{\mathbf{p}}_{0}, \overline{\mathbf{p}}_{0}\right], \mathbf{v}(\cdot) \in \mathcal{V}$ and $t \in\left[t_{0}, t_{N}\right]$.

A sufficient condition for cooperativity is that all offdiagonal components of $\partial \mathbf{f} / \partial \mathbf{x}$ are non-negative for any $\mathbf{x} \in \mathbb{D}, \mathbf{p} \in\left[\underline{\mathbf{p}}_{0}, \overline{\mathbf{p}}_{0}\right], \mathbf{v}(\cdot) \in \mathcal{V}$ and $t \in\left[t_{0}, t_{N}\right]$, (Smith, 1995).

All donor-controlled compartmental models (Jacquez, 1972), for instance, are cooperative, even if the flow of material is nonlinear.

More general classes of models may be considered, as in (Gennat and Tibken, 2004), by using results of (Müller, 1926). Note that the particular case of cooperative dynamics was already considered in (Müller, 1926).

As we shall see, these results make it possible to enclose the predicted set $\mathbb{X}_{t_{k+1} \mid t_{k}}$ at time $t_{k+1}$ in a subpaving, and thus to get a much more accurate set estimate than with an interval observer and for a larger class of models. We shall first present some slightly adapted versions of Müller's theorems for cooperative and uncooperative models. We shall then show how these theorems can be used to get inclusion functions for the solutions of (1).

\subsection{Müller's theorems}

These theorems provide existence and uniqueness results for the solutions of systems of ODEs within compact domains. They are reformulated here in a form suitable for state estimation.
Theorem 1. Let $\mathbf{f}(\mathbf{x}, \mathbf{p}, \mathbf{v}, t)$ be a continuous function over a domain $\mathbb{T}$ defined by

$$
\mathbb{T}:\left\{\begin{array}{l}
\boldsymbol{\omega}(t) \leqslant \mathbf{x} \leqslant \boldsymbol{\Omega}(t) \\
\underline{\mathbf{p}}_{0} \leqslant \mathbf{p} \leqslant \overline{\mathbf{p}}_{0} \\
\underline{\mathbf{v}}_{t_{0}}(t) \leqslant t \leqslant \mathbf{v}_{N}(t) \leqslant \overline{\mathbf{v}}(t)
\end{array}\right.
$$

with $\omega_{i}(t)$ and $\Omega_{i}(t)$ continuous functions over $\left[t_{0}, t_{N}\right]$ for all $i=1 \ldots n_{x}$ and such that

(1) $\boldsymbol{\omega}\left(t_{0}\right)=\underline{\mathbf{x}}_{0}$ and $\boldsymbol{\Omega}\left(t_{0}\right)=\overline{\mathbf{x}}_{0}$,

(2) the left derivatives $D^{-} \omega_{i}(t)$ and $D^{-} \Omega_{i}(t)$, and right derivatives $D^{+} \omega_{i}(t)$ and $D^{+} \Omega_{i}(t)$ of $\omega_{i}(t)$ and $\Omega_{i}(t)$ satisfy for $i=1 \ldots n_{x}$

$$
\begin{aligned}
& D^{ \pm} \omega_{i}(t) \leqslant \min _{\mathbb{T}_{i}(t)} f_{i}(\mathbf{x}, \mathbf{p}, \mathbf{v}, t), \\
& D^{ \pm} \Omega_{i}(t) \geqslant \max _{\overline{\mathbb{T}}_{i}(t)} f_{i}(\mathbf{x}, \mathbf{p}, \mathbf{v}, t),
\end{aligned}
$$

where $\mathbb{T}_{i}(t)$ is the subset of $\mathbb{T}$ defined by

$$
\underline{\mathbb{T}}_{i}(t):\left\{\begin{array}{l}
x_{i}=\omega_{i}(t) \\
\omega_{j}(t) \leqslant x_{j} \leqslant \Omega_{j}(t), j \neq i \\
\underline{\mathbf{p}}_{0} \leqslant \mathbf{p} \leqslant \overline{\mathbf{p}}_{0} \\
\underline{\mathbf{v}}(t) \leqslant \mathbf{v}(t) \leqslant \overline{\mathbf{v}}(t) \\
t=t
\end{array}\right.
$$

and where $\overline{\mathbb{T}}_{i}(t)$ is the subset of $\mathbb{T}$ defined by

$$
\overline{\mathbb{T}}_{i}(t):\left\{\begin{array}{l}
x_{i}=\Omega_{i}(t) \\
\omega_{j}(t) \leqslant x_{j} \leqslant \Omega_{j}(t), j \neq i \\
\underline{\mathbf{p}}_{0} \leqslant \mathbf{p} \leqslant \overline{\mathbf{p}}_{0} \\
\underline{\mathbf{v}}(t) \leqslant \mathbf{v}(t) \leqslant \overline{\mathbf{v}}(t) \\
t=t
\end{array}\right.
$$

Then, for any $\mathbf{x}_{0} \in\left[\underline{\mathbf{x}}_{0}, \overline{\mathbf{x}}_{0}\right], \mathbf{p} \in\left[\underline{\mathbf{p}}_{0}, \overline{\mathbf{p}}_{0}\right]$ and $\mathbf{v}(\cdot) \in$ $\mathcal{V},(1)$ admits a solution that stays in the domain

$$
\mathbb{E}:\left\{\begin{array}{l}
t_{0} \leqslant t \leqslant t_{N} \\
\boldsymbol{\omega}(t) \leqslant \mathbf{x} \leqslant \boldsymbol{\Omega}(t)
\end{array}\right.
$$

and takes the value $\mathbf{x}_{0}$ at $t_{0}$. If, moreover, for any $\mathbf{p} \in\left[\mathbf{p}_{0}, \overline{\mathbf{p}}_{0}\right]$ and $\mathbf{v}(\cdot) \in \mathcal{V}$, the function $\mathbf{f}(\mathbf{x}, \mathbf{p}, \mathbf{v}, t)$ is Lipschitzian with respect to $\mathbf{x}$ over $\mathbb{D}$, then this solution is unique for any given $\mathbf{p}$ and $\mathbf{v}(\cdot)$.

A useful variant of Theorem 1 can be obtained when the model is cooperative.

Theorem 2. Let $\mathbf{f}(\mathbf{x}, \mathbf{p}, \mathbf{v}, t)$ in (1) be a continuous function with the same domain $\mathbb{T}$ and constraints as in Theorem 1, but now with

$$
\underline{\mathbb{T}}_{i}(t)=\{\boldsymbol{\omega}(t)\} \times\left[\underline{\mathbf{p}}_{0}, \overline{\mathbf{p}}_{0}\right] \times[\underline{\mathbf{v}}(t), \overline{\mathbf{v}}(t)] \times\{t\}
$$

and

$$
\overline{\mathbb{T}}_{i}(t)=\{\boldsymbol{\Omega}(t)\} \times\left[\underline{\mathbf{p}}_{0}, \overline{\mathbf{p}}_{0}\right] \times[\underline{\mathbf{v}}(t), \overline{\mathbf{v}}(t)] \times\{t\} .
$$

If the model (1) is cooperative, then for any $\mathbf{x}_{0} \in$ $\left[\underline{\mathbf{x}}_{0}, \overline{\mathbf{x}}_{0}\right], \mathbf{p} \in\left[\mathbf{p}_{0}, \overline{\mathbf{p}}_{0}\right]$ and $\mathbf{v}(\cdot) \in \mathcal{V},(1)$ admits a solution that stays in $\mathbb{E}$ and takes the value $\mathbf{x}_{0}$ at $t_{0}$. This solution is unique under the same condition as in Theorem 1 .

Theorems 1 and 2 make it possible to bracket the solutions of (1) provided that functions $\boldsymbol{\omega}(t)$ and 
$\boldsymbol{\Omega}(t)$ can be found with the required properties. If such is the case, the interval function

$$
[\phi](t)=[\boldsymbol{\omega}(t), \boldsymbol{\Omega}(t)]
$$

is an inclusion function for all the solutions of (1).

Remark 1. The conditions required for the functions $\boldsymbol{\omega}(\cdot)$ and $\boldsymbol{\Omega}(\cdot)$ by Theorem 2 are less restrictive than in the case of Theorem 1 . Indeed, any couple of functions $(\boldsymbol{\omega}(\cdot), \boldsymbol{\Omega}(\cdot))$ that satisfies (6) and (7) satisfies also the corresponding conditions of Theorem 2 . Therefore, when the function $\mathbf{f}(\mathbf{x}, \mathbf{p}, \mathbf{v}, t)$ satisfies the cooperativity conditions of the second part of Theorem 2, the bracketing $[\phi](t)$ that can be obtained via this theorem will be at least as accurate as that obtained via Theorem 1.

\subsection{Obtaining $\boldsymbol{\omega}(\cdot)$ and $\boldsymbol{\Omega}(\cdot)$}

The main difficulty is to obtain suitable functions $\boldsymbol{\omega}(\cdot)$ and $\boldsymbol{\Omega}(\cdot)$. Explicit expressions are not trivial to come by in the general case, and this is why we shall consider particular cases where $\boldsymbol{\omega}(\cdot)$ and $\boldsymbol{\Omega}(\cdot)$ are easily obtained.

Assume first that it is possible to build a differential system

$\left\{\begin{array}{l}\underline{\mathbf{x}}^{\prime}=\underline{\mathbf{g}}_{1}\left(\underline{\mathbf{x}}, \overline{\mathbf{x}}, \underline{\mathbf{p}}_{0}, \overline{\mathbf{p}}_{0}, \underline{\mathbf{v}}(t), \overline{\mathbf{v}}(t), t\right), \underline{\mathbf{x}}\left(t_{0}\right)=\underline{\mathbf{x}}_{0}, \\ \overline{\mathbf{x}}^{\prime}=\overline{\overline{\mathbf{g}}}_{1}\left(\underline{\mathbf{x}}, \overline{\mathbf{x}}, \underline{\mathbf{p}}_{0}, \overline{\mathbf{p}}_{0}, \underline{\mathbf{v}}(t), \overline{\mathbf{v}}(t), t\right), \overline{\mathbf{x}}\left(t_{0}\right)=\overline{\mathbf{x}}_{0},\end{array}\right.$

the solution $\left(\boldsymbol{\omega}_{1}^{\mathrm{T}}(\cdot), \boldsymbol{\Omega}_{1}^{\mathrm{T}}(\cdot)\right)^{\mathrm{T}}$ of which satisfies the requirements of Theorem 1 . We shall see later how $\mathbf{g}_{1}$ and $\bar{g}_{1}$ can be built from $\mathbf{f}$. This set of two coupled ODEs involves no uncertain quantity so guaranteed numerical solutions can be obtained efficiently.

In the case of Theorem 2, one is led to consider instead the two following ODEs:

$$
\begin{aligned}
& \underline{\mathbf{x}}^{\prime}=\underline{\mathbf{g}}_{2}\left(\underline{\mathbf{x}}, \underline{\mathbf{p}}_{0}, \overline{\mathbf{p}}_{0}, \underline{\mathbf{v}}(t), \overline{\mathbf{v}}(t), t\right), \underline{\mathbf{x}}\left(t_{0}\right)=\underline{\mathbf{x}}_{0} \\
& \overline{\mathbf{x}}^{\prime}=\overline{\mathbf{g}}_{2}\left(\overline{\mathbf{x}}, \underline{\mathbf{p}}_{0}, \overline{\mathbf{p}}_{0}, \underline{\mathbf{v}}(t), \overline{\mathbf{v}}(t), t\right), \overline{\mathbf{x}}\left(t_{0}\right)=\overline{\mathbf{x}}_{0}
\end{aligned}
$$

such that for all $t \in\left[t_{0}, t_{N}\right], \mathbf{x} \in \mathbb{D}, \mathbf{p} \in\left[\underline{\mathbf{p}}_{0}, \overline{\mathbf{p}}_{0}\right]$ and $\mathbf{v} \in[\underline{\mathbf{v}}(t), \overline{\mathbf{v}}(t)]$ one has

$$
\underline{\mathbf{g}}_{2}\left(\mathbf{x}, \underline{\mathbf{p}}_{0}, \overline{\mathbf{p}}_{0}, \underline{\mathbf{v}}(t), \overline{\mathbf{v}}(t), t\right) \leqslant \mathbf{f}(\mathbf{x}, \mathbf{p}, \mathbf{v}, t)
$$

and

$$
\overline{\mathbf{g}}_{2}\left(\mathbf{x}, \underline{\mathbf{p}}_{0}, \overline{\mathbf{p}}_{0}, \underline{\mathbf{v}}(t), \overline{\mathbf{v}}(t), t\right) \geqslant \mathbf{f}(\mathbf{x}, \mathbf{p}, \mathbf{v}, t) .
$$

The solutions $\boldsymbol{\omega}_{2}(\cdot)$ and $\boldsymbol{\Omega}_{2}(\cdot)$ of these two ODEs then satisfy the conditions required by Theorem 2 . These two decoupled ODE's involve no uncertain quantity so guaranteed numerical solutions can again be obtained efficiently.

Example 1. When (1) can be written as

$$
\mathbf{x}^{\prime}=\mathbf{f}_{0}(\mathbf{x}, \mathbf{p}, t)+\mathbf{v}(t)
$$

and when the components $f_{0, i}(\mathbf{x}, \mathbf{p}, t), i=1 \ldots n_{x}$ of $\mathbf{f}_{0}(\mathbf{x}, \mathbf{p}, t)$ are monotonic with respect to $\mathbf{p}$ and to all components of $\mathbf{x}$ but $x_{i}$, the functions $\underline{\mathbf{g}}_{1}, \overline{\mathbf{g}}_{1}, \underline{\mathbf{g}}_{2}$ and $\overline{\mathbf{g}}_{2}$ are easy to define. For example, to build $\underline{g}_{1, i}$, in the formal expression of $f_{0, i}(\mathbf{x}, \mathbf{p}, t)$, replace

(1) $x_{i}$ by $\underline{x}_{i}$,

(2) for $j \neq i, x_{j}$ by $\bar{x}_{j}$ if $\frac{\partial f_{0, i}}{\partial x_{j}} \leqslant 0$ and by $\underline{x}_{j}$ if $\frac{\partial f_{0, i}}{\partial x_{j}} \geqslant 0$ for all $t \in\left[t_{0}, t_{N}\right], \mathbf{x} \in \mathbb{D}$ and $\mathbf{p} \in\left[\underline{\mathbf{p}}_{0}, \overline{\mathbf{p}}_{0}\right]$,

(3) for $k=1 \ldots n_{p}, p_{k}$ by $\bar{p}_{k}$ if $\frac{\partial f_{0, i}}{\partial p_{k}} \leqslant 0$ for all $t \in\left[t_{0}, t_{N}\right], \mathbf{x} \in \mathbb{D}$ and $\mathbf{p} \in\left[\underline{\mathbf{p}}_{0}, \overline{\mathbf{p}}_{0}\right]$ and by $\underline{p}_{k}$ if $\frac{\partial f_{0, i}}{\partial p_{k}} \geqslant 0$ for all $t \in\left[t_{0}, t_{N}\right], \mathbf{x} \in \mathbb{D}$ and $\mathbf{p} \in\left[\underline{\mathbf{p}}_{0}, \overline{\mathbf{p}}_{0}\right]$.

At last, add $\underline{v}_{i}(t)$ to the expression obtained. Reversed monotonicity conditions yields $\bar{g}_{1, i}$.

When no such monotonicity conditions are satisfied, a specific construction of the bounding system has to be considered. It may be helpful to split the time interval into smaller intervals on which monotonicity conditions are more easily satisfied (Marcelli and Rubbioni, 1997).

\section{EXAMPLES}

To compare results obtained by direct guaranteed numerical integration with those obtained using Müller's theorems, consider the two-compartment model of Figure 1. The parameters $k_{12}$ and $k_{21}$ are assumed to be constant. The parameter $k_{01}$ depends nonlinearly of the quantity of material in the first compartment, according to a Michaelis-Menten nonlinearity (Godfrey, 1983)

$$
k_{01}=\frac{p_{1}}{1+p_{2} x_{1}} .
$$

The parameter vector may thus be defined as $\mathbf{p}=$ $\left(p_{1}, p_{2}, k_{12}, k_{21}\right)^{\mathrm{T}}$. The quantities of material in the two compartments evolve according to

$$
\mathbf{x}^{\prime}=\left(\begin{array}{l}
-p_{4} x_{1}-\frac{p_{1} x_{1}}{1+p_{2} x_{1}}+p_{3} x_{2}+u \\
p_{4} x_{1}-p_{3} x_{2}
\end{array}\right)
$$

The initial state vector $\mathbf{x}_{0}=(1,0)$ is assumed to be known. For all $t \geqslant 0$, no input is considered, thus $u(t)=0$. Moreover, no state perturbation is considered. The parameter vector $\mathbf{p}$ is only assumed to belong to the (rather small) box

$\left[\mathbf{p}_{0}\right]=[0.9,1.1] \times[1.1,1.3] \times[0.45,0.55] \times[0.2,0.3]$.

Only the content of the second compartment is measured, thus $y_{\mathrm{m}}\left(t_{k}\right)=x_{2}\left(t_{k}\right)+w\left(t_{k}\right)$. The evolution of the state of $(11)$ is studied for $t \in[0,10]$.

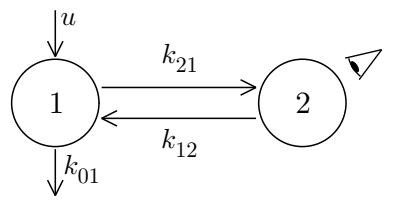

Fig. 1. Two-compartment model 


\subsection{Using only prediction}

Assume that no measurements are made, so SIVIA and IMAGESP are not used. Figure 2 presents the results obtained by direct guaranteed numerical integration of the uncertain system with the VNODE toolbox ${ }^{2}$ (Nedialkov and Jackson, 2001). The evolution of the enclosure for each component of $\mathbf{x}$ is in dotted lines. Direct numerical integration is unable to provide any solution for $t>2 \mathrm{~s}$.
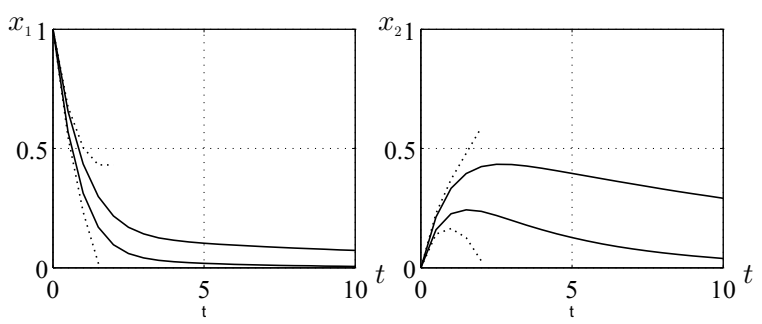

Fig. 2. Evolution of the state estimate using prediction only: direct numerical integration (dotted lines) and integration using cooperativity (bold lines)

Now, we use the fact that (11) is cooperative. As $\mathbf{x}$ and $\mathbf{p}$ are positive, the lower dynamical system

$$
\underline{\mathbf{x}}^{\prime}=\underline{\mathrm{g}}_{\mathrm{c}}\left(\underline{\mathbf{x}}, \underline{\mathbf{p}}_{0}, \overline{\mathbf{p}}_{0}, t\right), \underline{\mathbf{x}}(0)=\underline{\mathbf{x}}_{0}
$$

with

$$
\underline{\mathbf{g}}_{\mathbf{c}}(\cdot)=\left(\begin{array}{l}
-\bar{p}_{4} \underline{x}_{1}-\frac{\bar{p}_{1} \underline{x}_{1}}{1+\underline{p}_{2} \underline{x}_{1}}+\underline{p}_{3} \underline{x}_{2}+u \\
\underline{p}_{4} \underline{x}_{1}-\bar{p}_{3} \underline{x}_{2}
\end{array}\right)
$$

and the upper dynamical system

$$
\overline{\mathbf{x}}^{\prime}=\overline{\mathbf{g}}_{\mathrm{c}}\left(\overline{\mathbf{x}}, \underline{\mathbf{p}}_{0}, \overline{\mathbf{p}}_{0}, t\right), \overline{\mathbf{x}}(0)=\overline{\mathbf{x}}_{0}
$$

with

$$
\overline{\mathbf{g}}_{\mathrm{c}}(\cdot)=\left(\begin{array}{l}
-\underline{p}_{4} \bar{x}_{1}-\frac{\underline{p}_{1} \bar{x}_{1}}{1+\bar{p}_{2} \bar{x}_{1}}+\bar{p}_{3} \bar{x}_{2}+u \\
\bar{p}_{4} \bar{x}_{1}-\underline{p}_{3} \bar{x}_{2}
\end{array}\right)
$$

are such that $\underline{\mathbf{g}}_{\mathrm{c}}$ and $\overline{\mathbf{g}}_{\mathrm{c}}$ satisfy (9) and (10). Moreover, since $\underline{\mathbf{x}}_{0}=\overline{\mathbf{x}}_{0}=\mathbf{x}_{0}$, it is easy to check that the conditions of Theorem 2 hold. The solutions of (11) remain thus bounded between those of (12) and (13). Both solutions, again computed with VNODE, have been represented on Figure 2 in bold lines. Now, state estimates are provided for all $t \in[0,10]$.

\subsection{Taking measurements into account}

Assume now that measurements are available. Two state estimators will be considered. They only differ by how prediction is performed using IMAGESP. In the first case, an inclusion function for the solution of (11) is obtained by direct numerical integration (SEDNI) and in a second case, Müller's theorem is used (SE-MT).

\footnotetext{
2 http://www.cas.mcmaster.ca/ nedialk/Software/ VNODE/VNODE.shtml
}

Noise-free data have been obtained every $2 \mathrm{~s}$ as the output of a model with $\mathbf{p}$ at the center of $\left[\mathbf{p}_{0}\right]$. These data have then been corrupted by additive noise uniformly distributed in $[-0.05,0.05]$. This interval has also been taken for each $\left[w_{k}\right]$. Table 1 summarizes the measurement data.

Table 1. Noisy data for state estimation

\begin{tabular}{c|c|c|c|c|c}
$t_{k}$ & 2 & 4 & 6 & 8 & 10 \\
\hline$y\left(t_{k}\right)$ & 0.323 & 0.278 & 0.145 & 0.186 & 0.079
\end{tabular}

Figure 3 presents the enclosures obtained by both state estimation algorithms. Better results have been obtained using the estimator based on Müller's theorem. Computing time is about $70 \mathrm{~s}$ for SE-DNI and about $10 \mathrm{~s}$ for SE-MT, which is an additional advantage of the new approach.
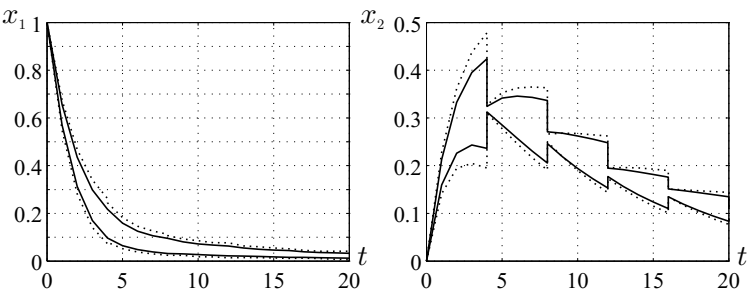

Fig. 3. Comparison of two state estimation algorithms: SE-DNI (dotted lines) and SE-MT (bold line)

\subsection{Joint parameter and state estimation}

To illustrate the use of Theorem 1, we focus now on joint parameter and state estimation. Assume that $p_{4}$ is added to $\mathbf{x}$ to form an extended state vector

$$
\mathbf{x}_{\mathrm{e}}=\left(\mathbf{x}^{\mathrm{T}}, p_{4}\right)^{\mathrm{T}} \text {. }
$$

As $p_{4}$ is assumed to be constant, the extended state satisfies

$$
\mathbf{x}_{\mathrm{e}}^{\prime}=\left(\begin{array}{l}
-x_{\mathrm{e} 3} x_{\mathrm{e} 1}-\frac{p_{1} x_{\mathrm{e} 1}}{1+p_{2} x_{\mathrm{e} 1}}+p_{3} x_{\mathrm{e} 2}+u \\
x_{\mathrm{e} 3} x_{\mathrm{e} 1}-p_{3} x_{\mathrm{e} 2} \\
0
\end{array}\right),
$$

with $\underline{\mathbf{x}}_{\mathrm{e}, 0}^{\mathrm{T}}=\left(\underline{\mathbf{x}}_{0}^{\mathrm{T}}, \underline{p}_{4}\right)$ and $\overline{\mathbf{x}}_{\mathrm{e}, 0}^{\mathrm{T}}=\left(\overline{\mathbf{x}}_{0}^{\mathrm{T}}, \bar{p}_{4}\right)$. The new parameter vector is now

$$
\mathbf{q}=\left(p_{1}, p_{2}, p_{3}\right)^{\mathrm{T}} \in\left(\left[p_{1}\right],\left[p_{2}\right],\left[p_{3}\right]\right)^{\mathrm{T}} .
$$

Cooperativity does not hold any more for (14), thus Theorem 2 cannot be used. However, a coupled pair of dynamical systems

$$
\left\{\begin{array}{l}
\underline{\mathbf{x}}_{\mathrm{e}}^{\prime}=\underline{\mathbf{g}}_{\mathrm{nc}}\left(\underline{\mathbf{x}}_{\mathrm{e}}, \overline{\mathbf{x}}_{\mathrm{e}}, \underline{\mathbf{q}}_{0}, \overline{\mathbf{q}}_{0}, t\right), \underline{\mathbf{x}}_{\mathrm{e}}(0)=\underline{\mathbf{x}}_{\mathrm{e}, 0}, \\
\overline{\mathbf{x}}_{\mathrm{e}}^{\prime}=\overline{\mathbf{g}}_{\mathrm{nc}}\left(\underline{\mathbf{x}}_{\mathrm{e}}, \overline{\mathbf{x}}_{\mathrm{e}}, \underline{\mathbf{q}}_{0}, \overline{\mathbf{q}}_{0}, t\right), \overline{\mathbf{x}}_{\mathrm{e}}(0)=\overline{\mathbf{x}}_{\mathrm{e}, 0},
\end{array}\right.
$$

with

$$
\underline{\mathrm{g}}_{\mathrm{nc}}(\cdot)=\left(\begin{array}{l}
-\bar{x}_{\mathrm{e} 3} \underline{x}_{\mathrm{e} 1}-\frac{\bar{p}_{1} \underline{x}_{\mathrm{e} 1}}{1+\underline{p}_{2} \underline{x}_{\mathrm{e} 1}}+\underline{p}_{3} \underline{x}_{\mathrm{e} 2}+u \\
\underline{x}_{\mathrm{e} 3} \underline{x}_{\mathrm{e} 1}-\bar{p}_{3} \underline{x}_{\mathrm{e} 2}
\end{array}\right)
$$


and

$$
\overline{\mathbf{g}}_{\mathrm{nc}}(\cdot)=\left(\begin{array}{l}
-\underline{x}_{\mathrm{e} 3} \bar{x}_{\mathrm{e} 1}-\frac{\underline{p}_{1} \bar{x}_{\mathrm{e} 1}}{1+\bar{p}_{2} \bar{x}_{\mathrm{e} 1}}+\bar{p}_{3} \bar{x}_{\mathrm{e} 2}+u \\
\bar{x}_{\mathrm{e} 3} \bar{x}_{\mathrm{e} 1}-\underline{p}_{3} \bar{x}_{\mathrm{e} 2} \\
0
\end{array}\right)
$$

can be built such that the conditions of Theorem 1 hold. The solution for (14) is thus bounded by the solutions for the two parts of (15).

Data have again been simulated on the same nominal system as before. The noise corrupting the measurement again belongs to the interval $[-0.05,0.05]$. For the sake of simplicity, all parameters (except $p_{4}$, put into the extended state vector) are assumed perfectly known. At $t=0, p_{4}$ is only known to belong to $[0.1,0.5]$.

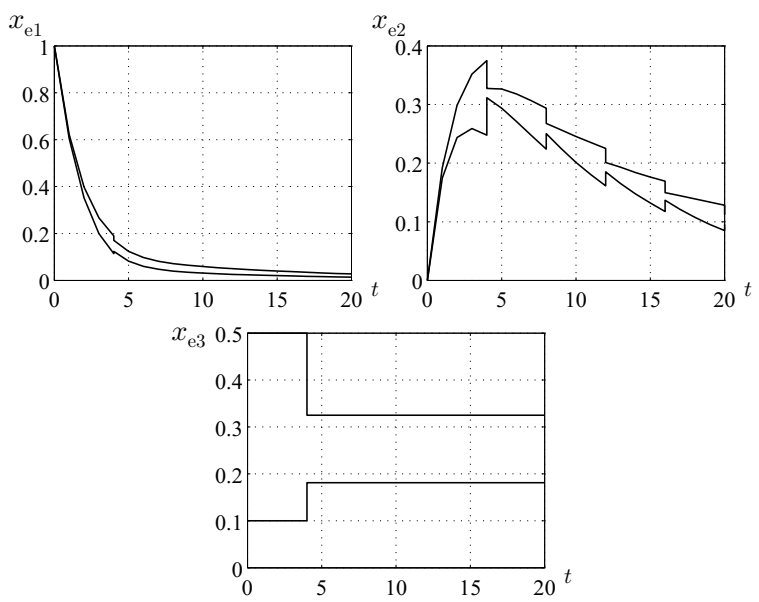

Fig. 4. Joint parameter and state estimation

The results for joint state and parameter estimation are displayed on Figure 4. Measurements allow a significant reduction of the uncertainty interval on $p_{4}$. This takes $40 \mathrm{~s}$ on an Athlon at $1.5 \mathrm{GHz}$.

\section{CONCLUSIONS}

The state enclosures provided by Theorems 1 and 2 allow guaranteed state estimation for a large class of systems described by uncertain ordinary differential equations. Joint parameter and state estimation may also be considered.

The main challenge is the struggle against the curse of dimensionality.

\section{REFERENCES}

Berz, M. and K. Makino (1998). Verified integration of ODEs and flows using differential algebraic methods on high-order Taylor models. Reliable Computing 4(4), 361-369.

Chernousko, F. L. (1994). State Estimation for Dynamic Systems. CRC Press. Boca Raton, FL.

Durieu, C., E. Walter and B. Polyak (2001). Multiinput multi-output ellipsoidal state bounding. Journal of Optimization Theory and Applications 111(2), 273-303.
Gennat, M. and B. Tibken (2004). Simulation of uncertain systems with guaranteed bounds. In: 11th GAMM - IMACS International Symposium on Scientific Computing, Computer Arithmetic, and Validated Numerics. Fukuoka, Japan.

Godfrey, K. (1983). Compartimental Models and Their Application. Academic Press. London.

Gouzé, J. L., A. Rapaport and Z. M. Hadj-Sadok (2000). Interval observers for uncertain biological systems. Journal of Ecological Modelling (133), 45-56.

Jacquez, J. A. (1972). Compartmental Analysis in Biology and Medicine. Elsevier. Amsterdam.

Jaulin, L. (2002). Nonlinear bounded-error state estimation of continuous-time systems. Automatica 38, 1079-1082.

Jaulin, L. and E. Walter (1993). Set inversion via interval analysis for nonlinear bounded-error estimation. Automatica 29(4), 1053-1064.

Jaulin, L., M. Kieffer, O. Didrit and E. Walter (2001). Applied Interval Analysis. Springer-Verlag. London.

Kieffer, M. and E. Walter (2005). Interval analysis for guaranteed nonlinear parameter and state estimation. Mathematical and Computer Modelling of Dynamic Systems 11(2), 171-181.

Kieffer, M., L. Jaulin and E. Walter (2002). Guaranteed recursive nonlinear state bounding using interval analysis. International Journal of Adaptative Control and Signal Processing 6(3), 193218.

Kurzhanski, A. and I. Valyi (1997). Ellipsoidal Calculus for Estimation and Control. Birkhäuser. Boston, MA.

Lohner, R. (1992). Computation of guaranteed enclosures for the solutions of ordinary initial and boundary value-problem. In: Computational Ordinary Differential Equations (J. R. Cash and I. Gladwell, Eds.). Clarendon Press. Oxford. pp. $425-435$.

Marcelli, C. and P. Rubbioni (1997). A new extension of classical Müller's theorem. Nonlinear Analysis 28, 1759-1767.

Müller, M. (1926). Über das Fundamentaltheorem in der Theorie der gewöhnlichen Differentialgleichungen. Math. Z. 26, 619-645.

Nedialkov, N. S. and K. R. Jackson (2001). Methods for initial value problems for ordinary differential equations. In: Perspectives on Enclosure Methods (U. Kulisch, R. Lohner and A. Facius, Eds.). Springer-Verlag. Vienna. pp. 219-264.

Raissi, T, N. Ramdani and Y. Candau (2004). Set membership state and parameter estimation for systems described by nonlinear differential equations. Automatica 40(10), 1771-1777.

Rapaport, A. and J.-L. Gouzé (2003). Parallelotopic and practical observers for nonlinear uncertain systems. Int. Journal of Control 76, 237-251.

Smith, H. L. (1995). Monotone Dynamical Systems: An Introduction to the Theory of Competitive and Cooperative Systems. Vol. 41 of Mathematical Surveys and Monographs. American Mathematical Society. Providence, RI. 